\title{
Indomethacin Suppresses Cisplatin-Enriched Stem-Like Breast Cancer Cells Through Modulating MicroRNAs Expression and Stimulating Antitumor Immunity
}

\author{
Norhan Mobark ${ }^{1}$, Nageh Elmahdy ${ }^{1}$, Mohamed Salem² ${ }^{2}$ Magda Elsayad ${ }^{1}$, and Abdel-aziz \\ Zidan $^{3}$ \\ ${ }^{1}$ Tanta University Faculty of Pharmacy \\ ${ }^{2}$ Tanta University Faculty of Science \\ ${ }^{3}$ Damanhour University Faculty of Science
}

May 27, 2020

\begin{abstract}
Background and Purpose: Cancer resistance to chemotherapy is a clinical dilemma that eventually leads to increased mortality. It is widely accepted that cancer stem cells (CSCs) have a pivotal role in the development of resistance. Nonsteroidal antiinflammatory drugs (NSAIDs) have shown a promise to combat CSCs, thus, we addressed for the first time the effect of indomethacin on cisplatin (CDDP)-resistant murine breast cancer along with the relevant mechanisms. Experimental Approach: The murine mammary adenocarcinoma, Ehrlich ascites carcinoma (EAC) cells, were made resistant by exposure to CDDP and the surviving cells were then analyzed by flow cytometry for the breast CSCs markers (CD44+CD24-). CDDP heavily enriched the CSCs population which was subsequently injected into mice. After induction of tumors, mice were treated with CDDP, or indomethacin, or co-treatment with both drugs, or left untreated. Upon termination of the treatment period, blood samples were collected to measure the percentage of CSCs markers (CD44, CD24, SCa-1) and the immune cells (CD4+, CD62L+, and $\mathrm{CD} 117+$ ). The tumors were excised and analyzed for the relative expression of drug resistance-mediating miRNAs (miR-7, miR21, miR-22, and miR-145) in addition to histopathological examination. Key Results: Indomethacin drastically diminished the tumorigenicity of CDDP-resistant cells along with enhancing its sensitivity to CDDP which were correlated with its suppressing ability of CD44+CD24- cells and manipulating effect on miRNAs expression. Besides, indomethacin expanded the pool of immune cells that impart antitumor response. Conclusion and Implications: Indomethacin through targeting CSCs may confer better outcome than conventional chemotherapeutics in the treatment of resistant breast cancer.
\end{abstract}

\section{Introduction}

Cancer is a complex disease featuring uncontrolled cellular growth resulting in the accumulation of abnormal cells [1]. Cancer is a leading cause of death worldwide with an estimated 9.6 million deaths in 2018. Globally, breast cancer $(\mathrm{BC})$ is the most common cancer in women with 2.1 million new cases were estimated in 2018 and it is the primary cause of cancer death among them as well [2]. In Egypt, $32 \%$ of the total cancer incidence rates among females were attributable to BC in 2013 which represented the most prevailing cancer across Egyptian women [3].

The great progress that has been made in cancer therapy is supposed to bring this cancer burden down, however, a considerable number of patients still experience relapse [4]. Albeit conventional therapies as chemotherapy and radiotherapy suppress tumor growth, the success of these agents is limited by therapyresistant cells which consequently recur the disease [5]. Accumulating evidence has suggested that tumors are 
made of a heterogeneous population of cells which include cancer stem cells (CSCs), a minority subpopulation of undifferentiated cells that generate the differentiated progeny that comprise the bulk of the tumor. CSCs are supposed to be the main culprit for tumor initiation, metastasis, chemotherapeutics resistance, and disease relapse [6].

In 2003, CSCs have been identified in a solid form of human breast cancer (BC) by M. Al-Hajj and M.S. Wicha [7]. Breast cancer stem cells (BCSCs) have been found to be characterized by CD44+ ${ }^{+}$D2 $4^{-/ \text {low }}$ the epithelial surface antigen $\left(\mathrm{ESA}^{+}\right)$phenotype [8]. As few as hundred $\mathrm{CD} 44^{+} / \mathrm{CD} 24^{-/ \text {low }} / \mathrm{ESA}^{+}$breast cancer cells were able to initiate tumor in vivo, unlike $\mathrm{CD} 44^{-} / \mathrm{CD} 24^{+}$breast cancer cells [6].

Unfortunately, standard therapies as chemotherapy, radiotherapy, and surgery are not only incapable of eradicating BCSCs, strikingly, BCSCs were further enriched after treatment of different breast cancer cell lines with different chemotherapeutic agents $[1,9]$. Similarly, there was an increase in the CD $44^{+} \mathrm{CD} 24^{-/ \text {low }}$ subpopulation in breast tumors harvested from doxorubicin-treated mice [9]. This entails looking for therapeutic agents that could target CSCs to completely eradicate cancer and prevent disease relapse.

Recently, nonsteroidal anti-inflammatory drugs (NSAIDs) have gained a big reputation for their chemopreventive effects against several cancer types including colorectal [10], breast [11], glioblastoma [12], esophageal [13], and gastric cancers [14]. Indomethacin, among them, has displayed to act as a chemosensitizer in resistant BC as well as reduce metastasis from human BC cells $[15,16]$. Since CSCs are linked to chemoresistance and metastasis, we postulated that the beneficial effects of indomethacin in BC could dwell in a CSC-suppressing effect. Herein, we investigated the anti-BCSC activity of indomethacin, particularly on chemotherapy-enriched CSCs.

\section{Materials and Methods}

\section{In vitro study}

\section{Cells and cell culture}

The murine mammary adenocarcinoma Ehrlich ascites carcinoma (EAC) cells were derived from Ehrlich ascites bearing female BALB/c mice (Pharmacology and Experimental Oncology Unit of the National Cancer Institute (NCI), Cairo, Egypt). The ascitic fluid was collected, suspended in PBS (Lonza, Verviers, Belgium), and centrifuged at $1200 \mathrm{rpm}$ for 10 minutes at $4{ }^{\circ} \mathrm{C}$ (ThermoFisher Scientific, Germany). Cells were cultured in Roswell Park Memorial Institute (RPMI) 1640 supplemented with 10\% FBS (Biowest, Nuaillé, France), 100 units/mL penicillin, $100 \mu \mathrm{g} / \mathrm{mL}$ streptomycin, $2 \mathrm{mM}$ L-glutamine (Lonza, Verviers, Belgium), and 7 $\mu \mathrm{L} / \mathrm{mL}$ amphotericin B (Lonza, Walkersville, MD USA). All cells were maintained in a $5 \% \mathrm{CO}_{2}$ incubator at $37^{\circ} \mathrm{C}$ (Nuaire, USA). To enrich CSCs, cultured cells were incubated till it reached $80 \%$ confluency and then exposed to a single concentration of either doxorubicin (DOX) $(2,4,6 \mu \mathrm{g} / \mathrm{mL}$ ), paclitaxel (PTX) (8, $12,16 \mu \mathrm{g} / \mathrm{mL}$ ), or cisplatin (CDDP) $(20,40,50 \mu \mathrm{g} / \mathrm{mL})$ for 72 hours. These concentrations were selected to broadly span concentrations above and below the IC50 of each drug in EAC cells which are as follows; DOX $\operatorname{IC50}=3 \mu \mathrm{g} / \mathrm{mL}$ [17], PTX IC50 $=12 \mu \mathrm{g} / \mathrm{mL}$ [18], and CDDP IC50 $=32 \mu \mathrm{g} / \mathrm{mL}$ [19].

\section{Drugs and antibodies}

Indomethacin was gifted from Sigma pharmaceutical company (Cairo, Egypt). CDDP, DOX, and PTX were purchased from Mylan (Pallini, Attiki, Greece), EIMC United Pharmaceuticals (Badr city, Cairo, A.R.E.), and Bristol-Myers Squibb (Latina, Italy), respectively. Antibodies used for flow cytometry were FITCconjugated anti-mouse/human CD44 and phycoerythrin (PE)-conjugated anti-mouse CD24 (BioLegend, San Diego, California, United States).

\section{Flow cytometric analysis}

To determine the concentration of chemotherapy that enriched CSCs the most, chemotherapy-treated EAC cells were detached by trypsin-EDTA (Biowest, Nuaillé, France) and suspended in PBS for flow cytometric analysis. FITC conjugated anti-CD44 and PE-conjugated anti-CD24 were added and incubated for 30 minutes in dark according to the manufacturer's instructions. Samples were then washed with PBS and analyzed 
using BD FACSCanto II flow cytometer (BD Biosciences, USA) coupled with a computer with BD FACS Diva software for data analysis.

\section{In vivo mouse experiments}

\section{Tumor models}

25-30 g female BALB/c mice were purchased from the National Research Center (Cairo, Egypt) and acclimated for 10 days. Animals were maintained and treated following the Research Ethics Committee-approved guidelines for the care and use of laboratory animals (College of Pharmacy, Tanta University, Egypt).

Based on the results of flow cytometry surface staining of chemotherapy-treated cells, $50 \mu \mathrm{g} / \mathrm{mL}$ CDDP was the concentration that induced the highest CSCs enrichment. Accordingly, this concentration was used for the subsequent in vivo experiment. The viable CDDP-treated cells were considered as being CDDPresistant cells. Either CDDP-resistant EAC cells or parent EAC cells were suspended in saline and counted via a Neubauer haemacytometer (Marienfeld, Germany) by the trypan blue (Lonza, Walkersville, MD USA) exclusion method under the EVOS XL Core inverted microscope AMEX1000 (Life Technologies, Carlsbad, California, USA).

CSCs in EAC were enriched by $50 \mu \mathrm{g} / \mathrm{mL}$ CDDP then $250 \times 10^{3}$ of CDDP-resistant cells were injected subcutaneously into the back of each mouse for a total of 28 mice, meanwhile, parent EAC cells were injected into another 28 mice $\left(250 \times 10^{3} \mathrm{EAC}\right.$ cells for each mouse). When palpable tumors developed, mice in each group were then randomly divided into 4 subgroups (control, CDDP, indomethacin, and the combination of the same dose and frequency of both drugs).

A single intraperitoneal dose of CDDP $(7.5 \mathrm{mg} / \mathrm{kg}$ body weight) was administered on the first day of treatment [20]. Indomethacin $(1.0 \mathrm{mg} / \mathrm{kg}$ body weight) was administered every day for 16 days as an oral suspension in a $0.5 \%$ carboxymethylcellulose (CMC) (Isochem, France) solution while on the same day both the control and CDDP alone-treated mice groups were given the same volume of $0.5 \%$ CMC solution without indomethacin [5].

Tumor size was measured by a Vernier caliper (APT, China) and tumor volume was calculated based on the formula: volume $=\left(\right.$ length $\times$ width $\left.^{2}\right) / 2$. The tumor growth rate was computed using the supplementary excel file provided by Gregory Hather, et al[21]. All mice were sacrificed on day 17 since the commencement of treatment and blood samples were collected in EDTA tubes for flow cytometric analysis of CSC and immunological markers. The tumor masses were dissected, washed with saline and weighed. Some of the excised tumors were immediately fixed in $10 \%$ buffered formalin solution for histopathological examination whereas the others were placed in $-80^{\circ} \mathrm{C}$ for $\mathrm{PCR}$ analysis.

\section{Flow cytometric analysis}

To investigate the CSC-suppressing effect as well as the immunomodulatory influence of indomethacin, flow cytometric analysis of CSC markers and the hematological and immunological markers was performed on the collected blood samples. Two panels of surface staining were made. Panel one included FITC-conjugated antiCD44 along with PE-conjugated rat anti-mouse stem cell antigen-1 (Sca-1). In panel two, PE-conjugated antiCD24, FITC-conjugated rat anti-mouse CD117, PerCP-conjugated rat anti-mouse CD4, and allophycocyanin (APC)-conjugated anti-mouse CD62L were added together. The samples were incubated in dark for $30 \mathrm{~min}$ before the lysing solution was added and then incubated for another 15 min in dark. PBS was added to wash the samples before the acquisition. Data analysis was made by BD FACS Diva software. The anti-SCa-1, anti-CD117, and anti-CD4 antibodies were all purchased from BD Biosciences (USA). The vendor for the anti-CD62L antibody was BioLegend (USA).

\section{Histopathology}

The formalin-fixed tumors were routinely processed in ascending grades of alcohol then xylene. The tissues were then embedded in paraffin blocks, serially sectioned to 3-5 $\mu \mathrm{m}$ thick before being stained with Hematoxylin and Eosin (H\&E, Sigma pharmaceutical company, Egypt). All stained tissue sections were examined 
under a light microscope (Olympus BX 51, Olympus America, Melville, USA) coupled with a digital camera (Olympus DP11) for photographing.

Quantitative reverse transcription PCR (qRT-PCR)

For microRNAs (miRNAs) expression assays, the tumor tissues were disrupted and homogenized using a TissueLyser II (QIAGEN, Germany) following the manufacturer's protocols. Total RNA was extracted using the RNeasy spin column and the concentration and purity of it were determined using a nanodrop (DS-11+ Spectrophotometer, Denovix, USA).

Using the TaqMan ${ }^{\circledR}$ MicroRNA Reverse Transcription Kit, cDNA was reverse transcribed using MultiScribeReverse Transcriptase by the thermal cycler (GeneAmp PCR System 9700, Applied Biosystems, Japan) following the TaqMan microRNA assays protocol. The PCR amplification step was done using TaqMan ${ }^{(\mathrm{r})} 2 \mathrm{X}$ Universal PCR Master Mix and TaqMan MicroRNA Assay (20X) in a QuantStudio 5 (Applied Biosystems, Thermo Fisher Scientific, Singapore). As an endogenous control, glyceraldehyde 3-phosphate dehydrogenase (GAPDH) was used to normalize the expression levels of target genes using the comparative $\mathrm{C}_{\mathrm{T}}$ method. Genes primers were purchased from OriGene Technologies (USA) except the reverse primer of miR-7 which was obtained from QIAGEN. The primers sequences are shown in Table 1 .

\subsection{Data and Statistical Analysis}

Statistical analysis was done using the Statistical Package for Social Science (SPSS) version 18. A difference between mean values is considered significant at $p<0.05$. The results were presented as mean $+-\mathrm{SD}$. All the figures were made by GraphPad Prism version 8.

\section{Results}

" $\Sigma^{\prime \prime} \varsigma \omega \varepsilon \rho \varepsilon \varepsilon \varphi \varphi \varepsilon \varsigma \tau \iota \varepsilon \lambda \psi \varepsilon \nu \rho \iota \zeta \eta \varepsilon \delta \beta \psi 50 \mu \gamma / \mu \Lambda " \Delta \Delta \Pi$

The percentage of $\mathrm{CD} 44^{+} \mathrm{CD} 24^{-}$cells was increased using different concentrations of DOX, CDDP, and PTX, however, the most significant enrichment was observed with $50 \mu \mathrm{g} / \mathrm{mL}$ CDDP $(50 \mu \mathrm{g} / \mathrm{mL}$ CDDP vs . EAC; $p=0.000000363$ ) (Fig. 1 ). This concentration was therefore selected for subsequent in vivo experiments.

\section{Tumorigenicity of CDDP-Resistant EAC Cells $v s$. Parent EAC Cells}

Based on the proportion of CD44 ${ }^{+} \mathrm{CD} 24^{-}$cells, CDDP-resistant cells had a higher proportion of CSCs than the parent EAC cells $(3.4 \%$ vs . 0.1\%). CDDP-resistant cancer cells showed a heightened ability to form tumors compared with the sensitive cells where the CDDP-resistant cells-derived tumors were initiated faster and significantly larger than their parent counterparts (Fig. 2A ).

On day 17 when the tumors were harvested, all the resistant cells-derived tumors were significantly larger than their corresponding sensitive tumors except for indomethacin where the difference between indomethacinreceiving resistant and sensitive tumors was not statistically significant $(p=0.44)$ (Fig. 2B ).

Indomethacin effectively and selectively reduced the tumorigenicity of CDDP-resistant cells in in vivo mouse experiments

Indomethacin in combination with CDDP decreased the tumor volumes in mice bearing parent SEC more significantly than CDDP (SEC vs . SEC-combination; $p=0.00015212$, SEC $v s$. SEC-CDDP; $p=0.00627464$ ), whereas indomethacin alone was not able to significantly reduce the tumor volumes (SEC vs. SECindomethacin; $p=0.143)$. In contrast, for CDDP-resistant tumors, indomethacin dramatically reduced the tumor volumes compared to CDDP alone or combination of both (SEC/CDDP vs. SEC/CDDPindomethacin; $p=0.00000037$, SEC/CDDP vs. SEC/CDDP-CDDP; $p=0.01159254$, SEC/CDDP vs. SEC/CDDP-combination; $p=0.00002975$ ) (Fig. 2 ).

The growth rates of the sensitive SEC tumors exceeded those of the resistant tumors 
The growth rates of the tumors were computed after the start of the treatment, unexpectedly, the growth rates of all the resistant tumors were lower than their analogous sensitive ones (Fig. 3A ). Noteworthily, the growth rates of the CDDP-resistant tumors were initially faster than the SEC tumors before the treatment has been commenced (growth rate of SEC/CDDP $=0.093952$ vs. $\mathrm{SEC}=0.058813$ ) (Fig. 3B ).

In a similar pattern to the tumor volumes, the combination of indomethacin and CDDP more effectively impeded the growth rates of the parent SEC tumors than either drug alone (SEC vs.SECcombination; $p=0.00005500$, SEC vs. SEC-CDDP; $p=0.00233442$, SEC vs. SEC-indomethacin; $p=$ $0.12394883)$, while the growth rates of the resistant tumors were slowed down the most by indomethacin (SEC/CDDP vs.SEC/CDDP-indomethacin; $p=0.00013892$, SEC/CDDP vs.SEC/CDDP-CDDP; $p=$ 0.04591864, SEC/CDDP vs.SEC/CDDP-combination; $p=0.00503803$ ) (Fig. 3A ).

\section{CDDP-resistant tumors exhibited extensively deregulated miRNAs expressions}

We determined the relative expressions of 4 miRNAs that are deregulated in murine breast cancer and involved in drug resistance. Compared with the untreated sensitive tumors, the untreated resistant tumors showed lower expressions of miR-7 and miR-145 besides overexpression of miR-21 and miR-22 ( $p=0.037$, 0.00024, 0.001, 0.0000136, respectively) (Fig. 4 ).

\section{MiRNAs Expressions are Altered in Response to Indomethacin}

$m i R-7$ expression was significantly increased by indomethacin (SEC vs . SEC-indomethacin; $p=2.17^{*} 10^{-21}$, SEC/CDDP vs . SEC/CDDP-indomethacin; $p=8.33^{*} 10^{-7}$ ). In contrast, the conventional chemotherapy further downregulated $m i R-7$ in both sensitive and resistant tumors, however, it did not reach statistical significance for the latter (SEC vs . SEC-CDDP; $p=0.037309067230362626$, SEC/CDDP vs . SEC/CDDPCDDP; $p=0.9029500148694073)$. Strikingly, indomethacin showed an ability to overcome the downregulatory effect of CDDP and even upregulate $m i R-7$ when both used together (SEC vs. SEC-combination; $p=$ 0.00000823, SEC/CDDP vs . SEC/CDDP-combination; $p=0.00129)$ (Fig. 4A ).

Unlike $m i R-7$, indomethacin reduced the expression of $m i R-145$ (SEC vs . SEC-indomethacin; $p=$ $0.000000057341, \mathrm{SEC} / \mathrm{CDDP}$ vs . SEC/CDDP-indomethacin; $p=0.0098)$ whereas the effect of CDDP was as the same as its effect on miR-7 (SEC vs . SEC-CDDP; $p=1.88^{*} 10^{-11}$, SEC/CDDP $v s$. SEC/CDDP$\mathrm{CDDP} ; p=0.000065)$. Unexpectedly, the combination of both indomethacin and CDDP downregulated $m i R-145$ to a less extent (SEC vs . SEC-combination; $p=0.0000000677$, SEC/CDDP $v s$. SEC/CDDPcombination; $p=0.046$ ) (Fig. 4B ).

For miR-21, indomethacin significantly downregulated its expression (SEC vs. SEC-indomethacin; $p$ $=0.029, \mathrm{SEC} / \mathrm{CDDP} v s$. SEC/CDDP-indomethacin; $p=0.032)$ while it has been overexpressed by CDDP (SEC vs . SEC-CDDP; $p=0.00000083$, SEC/CDDP $v s$. SEC/CDDP-CDDP; $p=0.000091$ ). The combination of both drugs has not significantly decreased the expression of miR-21 (SEC vs . SEC-combination; $p=0.058, \mathrm{SEC} / \mathrm{CDDP} v s$. SEC/CDDP-combination; $p=0.059)$ (Fig. 4C ).

The levels of miR-22 in tumor tissues were significantly reduced by CDDP, indomethacin, and the combination (SEC $v s$. SEC-CDDP; $p=0.024$, SEC/CDDP $v s$. SEC/CDDP-CDDP; $p=0.000125$ ) (SEC $v s$. SEC-indomethacin; $p=0.021$, SEC/CDDP vs . SEC/CDDP-indomethacin; $p=0.000027$ ) (SEC vs . SEC-combination; $p=0.008$, SEC/CDDP $v s$. SEC/CDDP-combination; $p=1.28^{*} 10^{-10}$ ) (Fig. 4D ).

Indomethacin negatively affected the stem cell markers and suppressed the CDDP-induced increase of $\mathrm{CD}^{+}{ }^{+} \mathrm{CD24}-$ cells

Indomethacin decreased the percentage of $\mathrm{CD} 44^{+}$cells $\left(p=7.9^{*} 10^{-10}\right)$ and increased that of CD24+ cells $\left(p=1.1^{*} 10^{-7}\right)$, whereas CDDP showed an opposite effect $\left(p=3.7^{*} 10^{-11}\right.$ and 0.003 for CD $44^{+}$and CD24 $4^{+}$, respectively), so as a result, the fraction of $\mathrm{CD} 44^{+} \mathrm{CD} 24^{-}$cells is considered to be gone up by CDDP unlike indomethacin. Strikingly, when indomethacin was used along with CDDP it has not only abolished the CDDP-associated elevation of CD44 ${ }^{+} \mathrm{CD} 24^{-}$cells, but the proportions were further dropped below control levels $\left(p=1.7^{*} 10^{-10}\right.$ and 0.019 for $\mathrm{CD} 44^{+}$and CD24 $4^{+}$, respectively) (Fig. 5 ). 
Both CDDP and indomethacin exerted a similar effect on the percentage of SCa- $1^{+}$cells where both drugs decreased the SCa- $1^{+}$population $\left(p=4.7^{*} 10^{-5}, 1.099^{*} 10^{-12}\right.$, and $1.088^{*} 10^{-12}$ for CDDP, indomethacin, and the combination, respectively) (Fig. 5 ).

\section{Indomethacin showed favorable responses for $\mathrm{CD} 2 \mathrm{~L}^{+}, \mathrm{CD}^{+}$, and $\mathrm{CD} 117^{+}$populations}

Indomethacin raised the percentages of $\mathrm{CD} 62 \mathrm{~L}^{+}, \mathrm{CD} 4^{+}$, and $\mathrm{CD} 117^{+}$cells $\left(p=1.08^{*} 10^{-12}, 1.08^{*} 10^{-12}\right.$, and $1.59^{*} 10^{-4}$, respectively) while a reverse effect was posed by CDDP, however, the decline was not statistically significant for $\mathrm{CD} 62 \mathrm{~L}^{+}$cells $\left(p=3.7^{*} 10^{-6}\right.$ and 0.009 for $\mathrm{CD}^{+}$and $\mathrm{CD} 117^{+}$cells, respectively). As expected, the combination treatment showed levels that are in between those of indomethacin and CDDP $\left(p=5.79^{*} 10^{-6}, 0.022\right.$, and 0.57 for $\mathrm{CD} 6 \mathrm{~L}^{+}, \mathrm{CD} 4^{+}$, and $\mathrm{CD} 117^{+}$cells, respectively) (Fig. 5 ).

\section{Histological Examination}

Upon examination of the $\mathrm{H} \& \mathrm{E}$ stained tumor sections, CDDP-resistant tumors were of a higher grade than the parent SEC tumors featuring extensive mitosis with pleomorphism and perineural invasion indicating the aggressiveness of such tumors. In the case of the parent SEC tumors, the three treatment modalities showed different degrees of necrosis with the highest extent of necrotic cells appeared with the co-treatment (Fig. 6 ). Remarkably, despite CDDP showed extensive areas of necrosis, there was a detectable tumor vascular embolus suggesting that CDDP did not target the metastasis-driving cells; CSCs (Fig. 6B2 ).

\section{Discussion and Conclusion}

Breast cancer patient's mortality is on the rise due to the lack of medications that could curb the activity of CSCs which drive distant metastasis and promote disease recurrence after chemotherapy, both of which are the primary causes of cancer-related deaths $[22,23]$. Current directions in cancer treatment aim to identify therapies that target CSCs thereby eliminating the roots of the disease.

NSAIDs have proven their chemoprophylactic effects in BC, and several studies have demonstrated that NSAIDs can even reduce the risk of BC recurrence [11]. NSAIDs reduced metastasis in cancer patients, of them, BC patients were included [24]. Since CSCs are the ones responsible for relapse and metastasis [6], we propose, based on these studies and our findings, that the therapeutic effects of NSAIDs might be ascribed to their anti-CSC effect.

Although there is extensive research on the CSC-inhibitory effect of NSAIDs, much of the research done investigated the modulation of stemness genes and epithelial mesenchymal transition (EMT) markers by NSAIDs as possible mechanisms for their CSC-suppressing ability [25-27] but little has been done on whether NSAIDs combat CSCs by affecting either miRNAs that are implicated in the drug resistance or the immune system whom CSCs can circumvent.

Herein, we aimed to examine the effect of indomethacin on two oncomirs and two tumor suppressor miRNAs in $\mathrm{BC}$ besides showcasing a little cue on the likely involvement of the antitumor immunity as a mechanism underlying the CSC-suppression mediated by indomethacin.

Much evidence indicate that CSCs subset markedly expands following chemotherapy [9] and we used this approach to enrich CSCs originally existing in EAC as these cells represent only a very small fraction of cancer population $(0.1 \%)$ which could have hindered our ability to either effectively induce resistant tumors or discover a potentially pronounced anti-CSC effect of the candidate drugs. However, the percentage of the CDDP-enriched CSCs was 3.4\% which is 34-fold higher than the portion in the parent EAC. As such, this CSCs-enriched population proved to be highly tumorigenic upon implantation in mice compared to their more differentiated progeny when an equivalent number of cells were injected and our result is in harmony with a previous report [28]. Intriguingly, although the tumors derived from the CDDP-resistant cells exhibited larger volumes, it grew slower than the parent tumors. These seemingly paradoxical behaviors could be potentially accredited to a relative shortage of oxygen and nutrients in quite big tumors which in turn retards the growth rate of such tumors [21]. 
Liu et al. studied the growth kinetics of BCSCs and proposed mathematical models thereafter. According to his study, non-stem cancer cells exert negative feedback on the proliferation rate and self-renewal ability of CSCs [29]. That could elaborate on why the antitumor effect of indomethacin on CDDP-resistant tumors was far more significant than the co-treatment. As CDDP preferentially targets the bulk tumor cells, it will be successful in letting the tumor sizes down, but meanwhile, the negative feedbacks on CSCs will be eliminated, therefore, CSCs will symmetrically proliferate at a high rate, and this is consistent with the dramatic increase in the CSCs fraction after CDDP treatment.

On the other hand, indomethacin selectively targets CSCs leaving the differentiated cancer cells and their negative feedbacks in action. As a result, the division of CSCs will be shifted towards an asymmetric one at a slower pace and this agrees well with the sharp drop in the CSCs population post indomethacin treatment. It is commonly believed that the optimum therapeutic strategy to achieve complete remission of cancer is to combine drugs that target both CSCs and non-CSCs. Based on our findings and the proposed models by Liu, Johnson et al. , the removal of the differentiated tumor cells will not make the outcome any better [29].

Concerning the more differentiated SEC tumors, although indomethacin has not significantly reduced their tumors sizes Liu, Johnson et al. , expected for such CSCs-targeted therapies to suppress tumor growth and inhibit relapse on the long term. On the contrary, relapse is probable with conventional chemotherapies despite the powerful fall in tumor size because of the accompanied CSCs enrichment [29]. This makes us recall the histological examination of SEC-CDDP tumors when we found a notable tumor vascular embolus indicating the advert of metastasis and subsequent relapse.

We then examined the relative expression of different miRNAs. BothmiR- 7 and $m i R-145$ were more downregulated in the CDDP-resistant tumors. A similar finding was observed in a study conducted by Pogribny et al. where miR-7 levels were 2.3 fold more downregulated in CDDP-resistant MCF-7 compared to MCF-7 cells [30]. Others reported that gall bladder cancer cells were sensitized to CDDP through miR-145-mediated regulation of MRP1 [31].

Contrarily, both miR-21 and miR-22 were overexpressed in SEC/CDDP tumors compared to the nonresistant ones. miR-21 was found to be involved in CDDP resistance through targeting the tumor suppressor phosphatase and tensin homolog (PTEN) in gastric cancer [32] and neuroblastoma and was overexpressed in CDDP-resistant cell lines [33]. In another study, the sensitivity of MCF-7 cells to CDDP was enhanced by knockdown of dicer which is accompanied by lower expression of miR-21. Collectively, these findings could explain the decreased sensitivity of the tumors derived from the CSCs-enriched population to CDDP treatment unlike the parent SEC tumors which responded better.

To examine whether indomethacin is capable of antagonizing the immunosuppressive impact of CDDP, we determined the levels of conventional blood and immune cells markers. CD4, CD117, and CD62L (otherwise known as L-selectin) are basic markers for T helper cells (Th) [34], hematopoietic stem cells (HSCs) [35], and white blood cells (WBCs) [36], respectively. CD4 is found on the surface of other immune cells such as dendritic cells [37] and natural killer T cells [38].

Although CDDP treatment has drastically shrunk the SEC tumors, its effect on the CDDP-resistant tumors was subtle. CDDP enriched $\mathrm{CD} 44^{+} \mathrm{CD} 24^{-}$cells and further downregulated miR-7 and $m i R-145$ while upregulatedmiR-21. The mechanisms by which CDDP modulated these miRNAs are still obscure as most of the studies examined the consequence of miRNAs expression pattern on the sensitivity of cancer to CDDP but none, to the best of our knowledge, have tested the effect of CDDP on miRNAs expression.

Moreover, CDDP decreased circulating $\mathrm{CD} 117^{+}, \mathrm{CD}_{2} \mathrm{~L}^{+}$, and $\mathrm{CD} 4^{+}$cells as well. A possible explanation for such deficiency is the well-known CDDP-prompted myelosuppression effect. A study conducted by Zhao et al. showed that CDDP-provoked $\mathrm{G} 2 / \mathrm{M}$ phase arrest in bone marrow cells resulting in bone marrow suppression [39].

On the other hand, albeit the SEC tumors sizes have not been significantly decreased by indomethacin, its effect on SEC/CPPD tumors was robust. Cells positive for CD44 or SCa-1 and those negative for CD24 
have been reduced by indomethacin, additionally, it promoted the expression of $m i R-7$ and declined that of miR-21 andmiR-22. Majumder et al . have found that COX-2 induces BCSCs through EP4-mediated $\mathrm{NOTCH} / \mathrm{WNT}$ axis and this effect was reversed with COX-2 inhibitor or EP4 antagonist [40]. This could clarify the indomethacin-associated decrease in CSCs markers. Furthermore, an earlier study demonstrated that pre-treatment of mammary cancer cells with COX-2 inhibitors caused suppression of radiotherapyinduced SCa- $1^{+}$cells [41] which supports the relevant finding of the indomethacin-accompanied reduction of $\mathrm{SCa}-1^{+}$pool.

Moreover, as miR-7 blocks the growth of CSCs in BC [42], it might explain the decrease of CD44+ CD24cells in indomethacin-treated mice due to its upregulatory effect on miR-7. Bourguignon et al . showed that miR-21 is induced in BC by binding of hyalouronan with CD44 [43], so miR-21 expression might be lowered in response to indomethacin due to the associated reduction in CD44 level. Consistently, it has been reported that the downregulation of $m i R$-21 stimulated the differentiation of chemo-resistant colon cancer and consequently enhanced the sensitivity to therapy [44]. Our findings provide an illustration of the enhanced susceptibility of the established tumors to CDDP when used simultaneously with indomethacin.

Of note, despite miR-145 is thought to be a tumor suppressor, it displayed a contradictory effect where it suppressed the differentiation of BCSCs via the $3^{6}$-untranslated region (3'-UTR) of insulin receptor substrate $1[45]$.

Furthermore, the percentages of cells positive for each of CD117, CD62L, and CD4 have been raised by indomethacin. Hoggatt et al.[46], have documented that NSAIDs can mobilize the HSCs from the bone marrow into the bloodstream. The mechanism of the NSAIDs mobilizing effect is based on inhibition of PGE2 synthesis which disrupts the production of osteopontin, a protein secreted by osteoblasts, that hooks the stem cells to the bone marrow. Since these HSCs give rise to all blood cell types, this might elucidate the indomethacin-mediated increase in blood cells expressing CD117, CD62L, CD4, and CD24. Considering this mobilizing effect, indomethacin therapy, unlike chemotherapy, can exclude the need for HSCs transplantation when used by cancer patients.

Aside from counteracting CDDP-resistance, indomethacin probably eradicates CSCs through immunostimulatory effects. It has been documented that PGE2 drives tumor progression by inducing myeloid-derived suppressor cells (MDSCs) and that EP antagonists block MDSCs differentiation [47]. MDSCs are immunosuppressive and they have been involved in BC promotion by conferring stem cell-like qualities as well as suppressing T-cell activation [48].

Interestingly, tumor-induced MDSCs downregulate the expression of L-selectin on $\mathrm{CD}^{+}$and $\mathrm{CD} 8^{+} \mathrm{T}$ cells impairing L-selectin dependent cell-mediated antitumor immunity [49] and this might explain the indomethacin-induced increase in $\mathrm{CD}_{2} \mathrm{~L}^{+}$cells. Furthermore, PGE2 reduces dendritic cells (DCs) differentiation and this type of immune cells is thought to eradicate CSCs [47, 50].

Intriguingly, the activity of the immune cells is influenced by miRNAs. For example, MDSCs highly express miR-21 which alters its differentiation and functional activity [51]. As indomethacin downregulates miR-21 , this could provide another support on how indomethacin might impede MDSCs activity. Moreover, miR21 downregulates the tumor suppressor programmed cell death-4 (PDCD4). PDCD4 inhibits the expression of the immunosuppressives IL-10 and IL-4 [52]. Both IL-4 and IL-10 promote CSCs survival [53, 54]. Furthermore, the expression of IL-12 is reciprocal to miR-21 [52] and unlike IL-10 and IL-4, IL-12 inhibits CSCs [55]. Hence, indomethacin may kill CSCs through its downregulatory impact onmiR-21 and the subsequent reduction in IL-4 and IL-10 as well as elevation of IL-12.

Additionally, it has been shown that PGE2 suppresses T cell proliferation [56], thus, the apparent increase in peripheral $\mathrm{CD}^{+}$population could be accounted for the PGE2 synthesis inhibition by indomethacin. Moreover, we can expect that indomethacin might augment Th1 actions as long as PGE2 suppresses Th1 responses [56]. Notably, both IL-4 and IL-10 inhibit Th1 cell production and function, respectively [57, 58] whereas IL-12 is indeed involved in Th1 polarization [52]. Given these, we could deduce that the indomethacin-associated increase in peripheral $\mathrm{CD} 4^{+}$cells is potentially in favor of Th1 cell type. 
In fact, recent studies have indicated that immunotherapeutic strategies in BC should be directed towards type 1 immune response that is rich in Th1 cells [59]. However, whether the indomethacin-associated CD4 ${ }^{+}$ cells are tumor-antigen specific or even have a role in fighting CSCs needs to be investigated. Interestingly, elevated levels of antigen specific $\mathrm{CD} 4^{+} \mathrm{T}$ cells in the peripheral blood of $\mathrm{BC}$ patients who undergo anticancer therapy is not unfamiliar. This is exemplified by trastuzumab therapy which boosted the levels of circulating HER2 specific CD4 ${ }^{+}$Th1 cells in BC patients who otherwise normally have few circulating antigen-specific $\mathrm{T}$ cells able to eradicate their tumors [59].

Impressively, one study discovered the existence of anti-CSC specific CD4 ${ }^{+} \mathrm{T}$ cells in the peripheral blood of ovarian cancer patients [60]. This encourages us to complement the current work by conducting further studies on the phenotypic and functional characterization of the indomethacin-induced $\mathrm{CD} 4^{+}$cells. Moreover, whether the high levels of $\mathrm{CD}^{+}$cells in the blood are correlated with the disease outcome remains to be explored. If this is the case, this could be harnessed as a prognostic tool instead of invasively monitoring the level of tumor-infiltrating lymphocytes.

There is also a link between miR-22 and the immune system, for instance, miR-22 is upregulated in DCs retarding their anti-tumor activity [61]. Thus, the indomethacin-induced downregulation of $m i R$-22 could promote the CSC-suppressing ability of tumor-associated DCs.

In conclusion, this study shows that indomethacin has a CSC-suppressing effect in BC and inhibits CDDPinduced increase of CSCs presumably through modulation of miRNAs implicated in drug resistance as well as triggering an antitumor immune response. To this end, NSAIDs sound to have far-reaching mechanisms behind its CSC-suppressing activity that necessitate deeper investigations to exactly unravel the pathways of such mechanisms.

\section{References}

1. Rundstadler, T., et al., Polypyridyl Zinc (II)-Indomethacin Complexes with Potent Anti-Breast Cancer Stem Cell Activity. Molecules, 2018. 23 (9): p. 2253.

2. Bray, F., et al., Global cancer statistics 2018: GLOBOCAN estimates of incidence and mortality worldwide for 36 cancers in 185 countries. CA Cancer J Clin, 2018. 68 (6): p. 394-424.

3. Ibrahim, A., et al., Cancer incidence in Egypt: results of the national population-based cancer registry program. J Cancer Epidemiol, 2014. 2014 : p. 437971.

4. Dawood, S., L. Austin, and M. Cristofanilli, Cancer stem cells: implications for cancer therapy. Oncology, 2014. 28 (12): p. 1101-1107.

5. Moon, C., et al., Nonsteroidal anti-inflammatory drugs suppress cancer stem cells via inhibiting PTGS2 (cyclooxygenase 2) and NOTCH/HES1 and activating PPARG in colorectal cancer. Int J Cancer, 2014.134 (3): p. 519-29.

6. Palomeras, S., S. Ruiz-Martinez, and T. Puig, Targeting breast cancer stem cells to overcome treatment resistance. Molecules, 2018.23 (9): p. E2193.

7. Al-Hajj, M., et al., Prospective identification of tumorigenic breast cancer cells. Proc Natl Acad Sci U S A, 2003. 100 (7): p. 3983-8.

8. Butti, R., et al., Breast cancer stem cells: biology and therapeutic implications. Int J Biochem Cell Biol, 2019. 107 : p. 38-52.

9. Jia, D., et al., Cardamonin reduces chemotherapy-enriched breast cancer stem-like cells in vitro and in vivo. Oncotarget, 2016.7 (1): p. 771-85.

10. Rigas, B. and G. Tsioulias, The evolving role of nonsteroidal anti-inflammatory drugs in colon cancer prevention: a cause for optimism. J Pharmacol Exp Ther, 2015. 353 (1): p. 2-8. 
11. Moris, D., et al., The role of NSAIDs in breast cancer prevention and relapse: current evidence and future perspectives.Breast Care (Basel), 2016. 11 (5): p. 339-344.

12. Allani, S., H. Weissbach, and M. Lopez Toledano, Sulindac induces differentiation of glioblastoma stem cells making them more sensitive to oxidative stress. Neoplasma, 2018. 65 (3): p. 376-388.

13. Qin, S., et al., Indomethacin induces apoptosis in the EC109 esophageal cancer cell line by releasing second mitochondria-derived activator of caspase and activating caspase-3. Mol Med Rep, 2015.11 (6): p. 4694-4700.

14. Akrami, H., et al., Ibuprofen reduces cell proliferation through inhibiting Wnt/beta catenin signaling pathway in gastric cancer stem cells. Cell Biol Int, 2018. 42 (8): p. 949-958.

15. Natarajan, K., et al., Exposure of human breast cancer cells to the anti-inflammatory agent indomethacin alters choline phospholipid metabolites and Nm23 expression. Neoplasia, 2002. 4 (5): p. 409-416.

16. Zhou, Y., et al., Doxorubicin-loaded redox-responsive micelles based on dextran and indomethacin for resistant breast cancer. Int J Nanomedicine, 2017. 12 : p. 6153-6168.

17. Sokar, S., et al., Pharmacokinetic and pharmacologic study of two P-glycoprotein modulating agents combined with doxorubicin. JPCS, 2012. 5 : p. 1-11.

18. Arican, G. and E. Arican, Evaluation of the apoptotic and antiproliferative activities of paclitaxel in Ehrlich ascites tumor cells. Biotechnology \& Biotechnological Equipment, 2006.20 (3): p. 69-75.

19. Verma, A. and S. Prasad, Bioactive component, cantharidin from Mylabris cichorii and its antitumor activity against Ehrlich ascites carcinoma. Cell Biol Toxicol, 2012. 28 (3): p. 133-147.

20. El-Naa, M., M. Othman, and S. Younes, Sildenafil potentiates the antitumor activity of cisplatin by induction of apoptosis and inhibition of proliferation and angiogenesis. Drug Des Devel Ther, 2016. 10 : p. 3661-3672.

21. Hather, G., et al., Growth rate analysis and efficient experimental design for tumor xenograft studies. Cancer Inform, 2014.13 (Suppl 4): p. 65-72.

22. Scully, O., et al., Breast cancer metastasis. Cancer Genomics Proteomics, 2012. 9 (5): p. 311-20.

23. Rawindraraj, A., C. Zhou, and V. Pathak, Delayed breast cancer relapse with pleural metastasis and malignant pleural effusion after long periods of disease-free survival. Respirol Case Rep, 2018.6 (9): p. e00375.

24. Zhao, X., Z. Xu, and $\mathrm{H}$. Li, NSAIDs use and reduced metastasis in cancer patients: results from a meta-analysis. Sci Rep, 2017.7 (1): p. 1875.

25. Huang, C., et al., Celecoxib targets breast cancer stem cells by inhibiting the synthesis of prostaglandin E2 and down-regulating the Wnt pathway activity. Oncotarget, 2017. 8 (70): p. 115254-115269.

26. Zhu, C., et al., Phosphosulindac (OXT-328) selectively targets breast cancer stem cells in vitro and in human breast cancer xenografts. Stem Cells, 2012. 30 (10): p. 2065-2075.

27. Tu, L., et al., Effect of aspirin on breast cancer stem cells and stemness of breast cancer. Zhonghua Yi Xue Za Zhi, 2018.98 (44): p. 3598-3602.

28. Fang, D., et al., Antitumor efficacy of the dual PI3K/mTOR inhibitor PF-04691502 in a human xenograft tumor model derived from colorectal cancer stem cells harboring a PIK3CA mutation. PLoS One, 2013. 8 (6): p. e67258.

29. Liu, X., et al., Nonlinear growth kinetics of breast cancer stem cells: implications for cancer stem cell targeted therapy. Sci Rep, 2013. 3 : p. 2473. 
30. Pogribny, I., et al., Alterations of microRNAs and their targets are associated with acquired resistance of MCF-7 breast cancer cells to cisplatin. Int J Cancer, 2010. 127 (8): p. 1785-1794.

31. Zhan, M., et al., miR-145 sensitizes gallbladder cancer to cisplatin by regulating multidrug resistance associated protein 1.Tumour Biol, 2016. 37 (8): p. 10553-10562.

32. Hu, W., et al., Functional miRNAs in breast cancer drug resistance. Onco Targets Ther, 2018. 11 : p. $1529-1541$.

33. Koturbash, I., et al., microRNAs as pharmacogenomic biomarkers for drug efficacy and drug safety assessment. Biomark Med, 2015.9 (11): p. 1153-1176.

34. Stubbington, M.J., et al., An atlas of mouse CD4(+) T cell transcriptomes. Biol Direct, 2015. 10 : p. 14.

35. Kyle-Cezar, F., et al., Expression of c-kit and Sca-1 and their relationship with multidrug resistance protein 1 in mouse bone marrow mononuclear cells. Immunology, 2007. 121 (1): p. 122-8.

36. Ivetic, A., Signals regulating L-selectin-dependent leucocyte adhesion and transmigration. Int J Biochem Cell Biol, 2013.45 (3): p. 550-5.

37. McLellan, A., et al., Anatomic location and T-cell stimulatory functions of mouse dendritic cell subsets defined by CD4 and CD8 expression. Blood, 2002. 99 (6): p. 2084-93.

38. Liao, C., M. Zimmer, and C. Wang, The functions of type I and type II natural killer T cells in inflammatory bowel diseases. Inflamm Bowel Dis, 2013. 19 (6): p. 1330-8.

39. Zhao, R., Q. Chen, and Y.-m. He, The effect of Ganoderma lucidum extract on immunological function and identify its anti-tumor immunostimulatory activity based on the biological network. Sci Rep, 2018. 8 (1): p. 12680 .

40. Majumder, M., et al., COX-2 induces breast cancer stem cells via EP4/PI3K/AKT/NOTCH/WNT axis. Stem Cells, 2016. 34 (9): p. 2290-2305.

41. Gong, J., et al., Genotoxic stress induces Sca-1-expressing metastatic mammary cancer cells. Mol Oncol, 2018. 12 (8): p. 1249-1263.

42. Okuda, H., et al., miR-7 suppresses brain metastasis of breast cancer stem-like cells by modulating KLF4. Cancer Res, 2013.73 (4): p. 1434-1444.

43. Bourguignon, L., et al., Hyaluronan-CD44 interaction with protein kinase C(epsilon) promotes oncogenic signaling by the stem cell marker Nanog and the Production of microRNA-21, leading to down-regulation of the tumor suppressor protein PDCD4, anti-apoptosis, and chemotherapy resistance in breast tumor cells. $\mathrm{J}$ Biol Chem, 2009.284 (39): p. 26533-46.

44. Vidyasekar, P., et al., Genome wide expression profiling of cancer cell lines cultured in microgravity reveals significant dysregulation of cell cycle and MicroRNA gene networks. PloS One, 2015.10 (8): p. e0135958.

45. Zhang, K., et al., MicroRNAs in the diagnosis and prognosis of breast cancer and their therapeutic potential. Int J Oncol, 2014.45 (3): p. 950-958.

46. Hoggatt, J., et al., Differential stem- and progenitor-cell trafficking by prostaglandin E2. Nature, 2013. 495 (7441): p. 365-9.

47. Sinha, P., et al., Prostaglandin E2 promotes tumor progression by inducing myeloid-derived suppressor cells. Cancer Res, 2007.67 (9): p. 4507-4513.

48. Peng, D., et al., Myeloid-derived suppressor cells endow stem-like qualities to breast cancer cells through IL6/STAT3 and NO/NOTCH cross-talk signaling. Cancer Res, 2016. 76 (11): p. 3156-3165. 
49. Hanson, E., et al., Myeloid-derived suppressor cells down-regulate L-selectin expression on CD4+ and CD8+ T cells. J Immunol, 2009. 183 (2): p. 937-44.

50. Nguyen, S., et al., Targeting specificity of dendritic cells on breast cancer stem cells: in vitro and in vivo evaluations. Onco Targets Ther, 2015. 8 : p. 323-34.

51. Curtale, G., MiRNAs at the crossroads between innate immunity and cancer: focus on macrophages. Cells, 2018. 7 (2): p. 12.

52. Kumarswamy, R., I. Volkmann, and T. Thum, Regulation and function of miRNA-21 in health and disease. RNA Biol, 2011.8 (5): p. 706-713.

53. Francipane, M., et al., Crucial role of interleukin-4 in the survival of colon cancer stem cells. Cancer Res, 2008. 68 (11): p. 4022-5.

54. Yang, L., et al., IL-10 derived from M2 macrophage promotes cancer stemness via JAK1/STAT1/NFkappaB/Notch1 pathway in non-small cell lung cancer. Int J Cancer, 2019. 145 (4): p. 1099-1110.

55. Xiang, L. and H. Liang, Interleukin-12 implication in tumor initiating capacity of colorectal cancer stem cells. Chemotherapy, 2016. 5 : p. 209.

56. Bruttel, V. and J. Wischhusen, Cancer stem cell immunology: key to understanding tumorigenesis and tumor immune escape? Front Immunol, 2014. 5 : p. 360.

57. Lazarski, C., et al., IL-4 attenuates Th1-associated chemokine expression and Th1 trafficking to inflamed tissues and limits pathogen clearance. PloS One, 2013. 8 (8): p. e71949.

58. Rutz, S., et al., Notch regulates IL-10 production by Thelper 1 cells. Proc Natl Acad Sci U S A, 2008. 105 (9): p. 3497-3502.

59. Disis, L. and E. Stanton, Immunotherapy in breast cancer: an introduction. Breast, $2018 . \mathbf{3 7}$ : p. 196-199.

60. Di, J., et al., Functional OCT4-specific CD4+ and CD8+ T cells in healthy controls and ovarian cancer patients. Oncoimmunology, 2013.2 (5): p. e24271.

61. Liang, X., et al., MicroRNA-22 impairs anti-tumor ability of dendritic cells by targeting p38. PLoS One, 2015. 10 (3): p. e0121510.

Table 1. Primers sequences used for qRT-PCR

\begin{tabular}{lll}
\hline Gene & Forward primer $\left(\mathbf{5}^{\mathbf{6}} \mathbf{3}^{\mathbf{6}}\right)$ & Reverse primer $\left.\mathbf{( 5}^{\mathbf{6}} \mathbf{- 3}\right)$ \\
\hline miR-7 & TGGAAGACTAGTGATTTTGTTGT & Universal primer (Qiagen) \\
$\mathbf{m i R - 2 1}$ & AGCTTATCAGACTGATGTTG & GAACATGTCTGCGTATCTC \\
$\mathbf{m i R - 2 2}$ & GTTCTTCAGTGGCAAGCTT & GAACATGTCTGCGTATCTC \\
miR-145 & CCAGTTTTCCCAGGAATCC & GAACATGTCTGCGTATCTC \\
GAPDH & GTCGGTGTGAACGGATTTG & GAATTTGCCGTGAGTGGAG \\
\hline
\end{tabular}

GAPDH $=$ glyceraldehyde 3-phosphate dehydrogenase.

Figures 


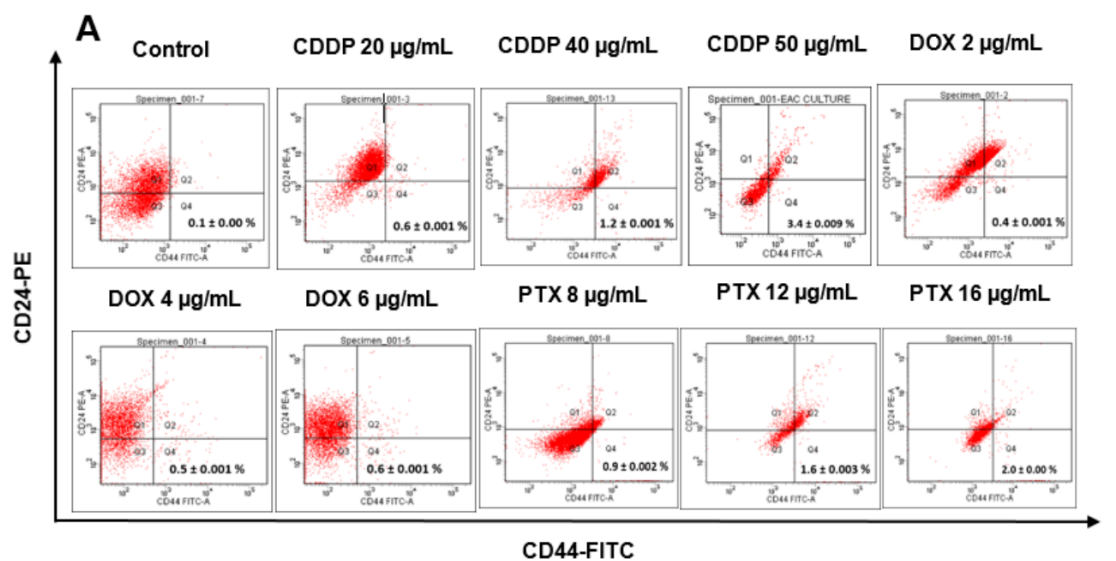

B

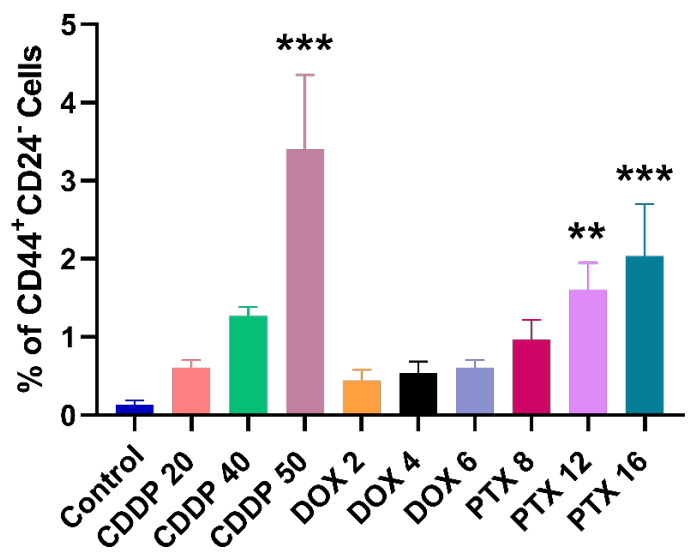

Fig. 1. Enrichment of CD44 ${ }^{+} \mathrm{CD}^{-} 4^{-}$cells within EAC cells using different concentrations of different chemotherapeutics . A. Dot plot of flow cytometry using FITC anti-CD44 and PE anti-CD24. Ehrlich cells were cultured in T-25 tissue culture flasks at a seeding density equals to $2 \times 10^{6}$ cells/flask. When the cells reached $80 \%$ confluency, various concentrations of CDDP, DOX, and PTX were added for 72 hrs. ( $\mathrm{n}=3$ for each concentration). The media was then discarded and adherent cells were washed by PBS and detached by trypsin-EDTA for 5-10 min and the latter was then inactivated by FBS. Cells were then collected in tubes and analyzed by flow cytometry for CSCs markers.B. Bar chart of the percentages of CD44 CD24cells of the various chemotherapeutics concentrations used showing the significance levels against control. CDDP at concentration $=50 \mu \mathrm{g} / \mathrm{mL}$ showed the highest enrichment of CSCs. One-way ANOVA was done. Data are presented as mean $\pm \mathrm{SD} .{ }^{* *} p<0.01$, ${ }^{* * *} p<0.001$. EAC: Ehrlich ascites carcinoma, FITC: fluorescein isothiocyanate, CD: cluster of differentiation, PE: phycoerythrin, PBS: phosphate-buffered saline, FBS: fetal bovine serum, CSCs: cancer stem cells, CDDP: cisplatin, DOX: doxorubicin, PTX: paclitaxel. 
A
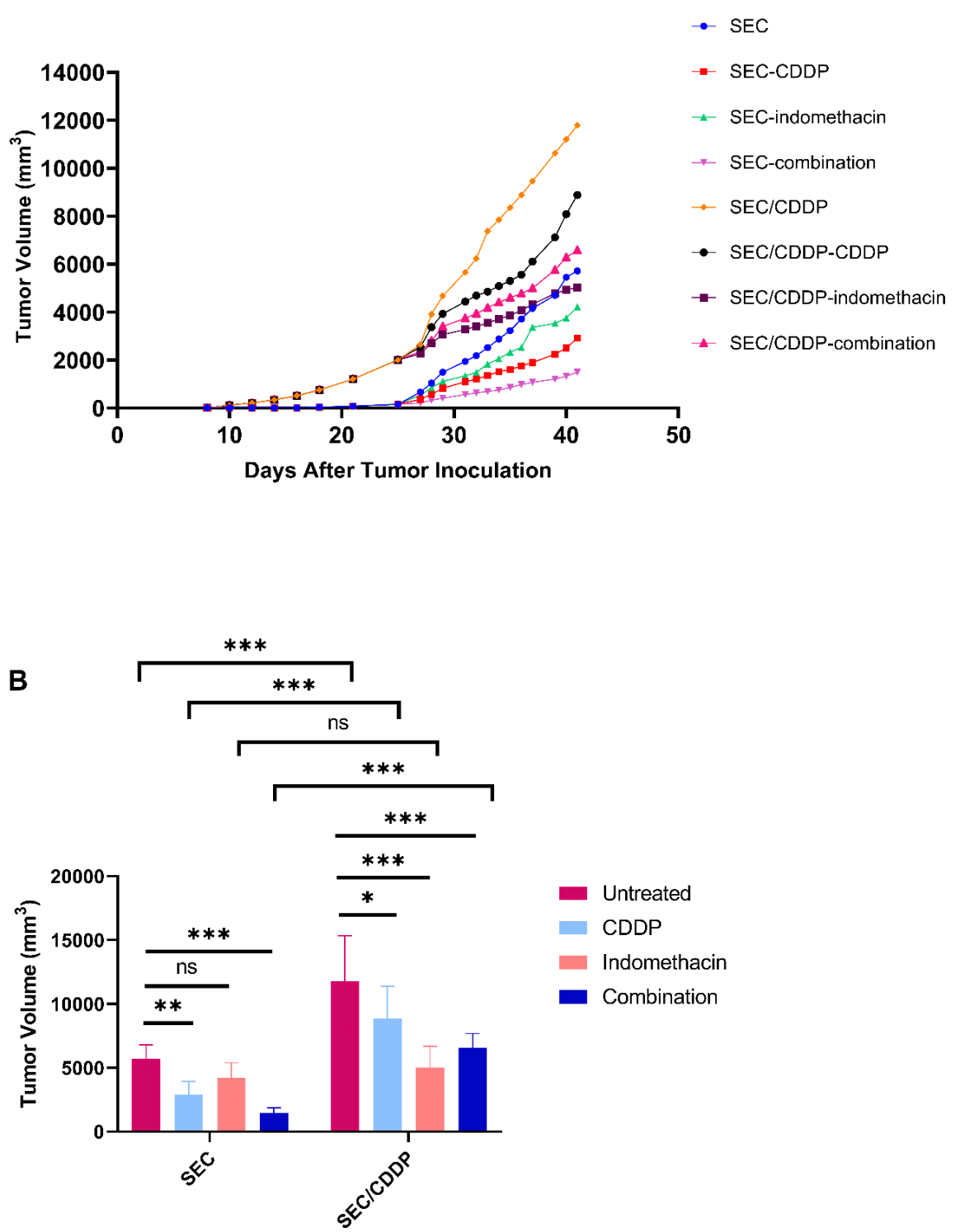

Fig. 2. Tumorigenicity of CDDP-resistant EAC cells vs. parent EAC cells. A. The tumor growth progress displaying the differential tumorigenicity between the resistant and sensitive EAC cells. B. Two-way ANOVA analysis was done for tumor size on day 17. Data are expressed as mean \pm SD. * $p<0.05,{ }^{* *} p<0.01,{ }^{* * *} p<0.001$, ns: nonsignificant. EAC: Ehrlich ascites carcinoma, SEC: solid Ehrlich carcinoma, SEC-CDDP: SEC treated with cisplatin, SEC-indomethacin: SEC treated with indomethacin, SEC-combination: SEC treated with CDDP and indomethacin, SEC/CDDP: cisplatin-resistant SEC, SEC/CDDP-CDDP: cisplatin-resistant SEC treated with cisplatin, SEC/CDDP-indomethacin: cisplatinresistant SEC treated with indomethacin, SEC/CDDP-combination: cisplatin-resistant SEC treated with CDDP and indomethacin. 


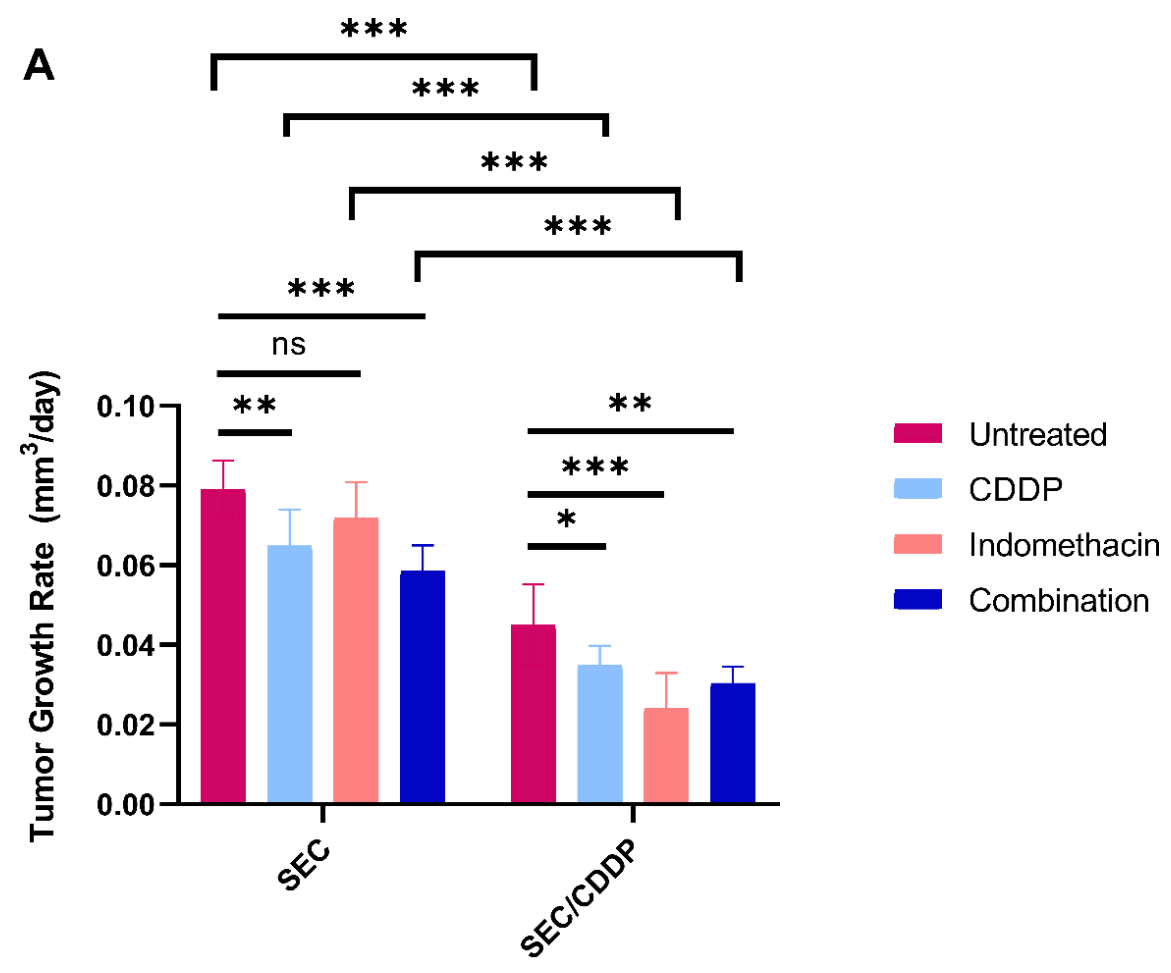

B

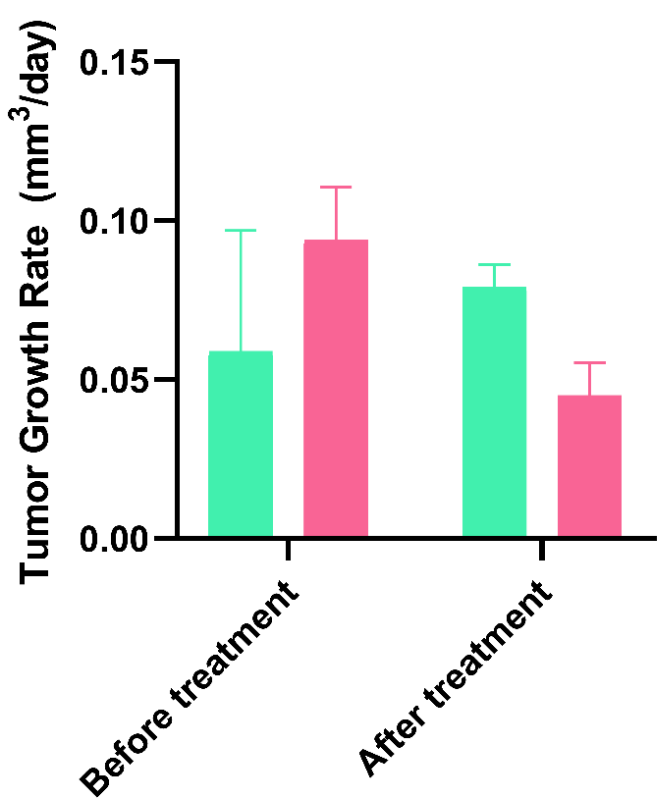

\section{SEC}

SEC/CDDP

Fig. 3. The tumor growth rates of the different studied groups. A. Tumor growth rates showing a higher rate for the parent SEC tumors. Two-way ANOVA analysis was done. Data are expressed as mean \pm SD.B. Tumor growth rates for SEC and SEC/CDDP tumors before and after kicking off the treatment. 
The growth rate of SEC/CDDP tumors was higher than the parental SEC tumors before the treatments were given. SEC: solid Ehrlich carcinoma, CDDP: cisplatin, SEC/CDDP: CDDP-resistant SEC.

A
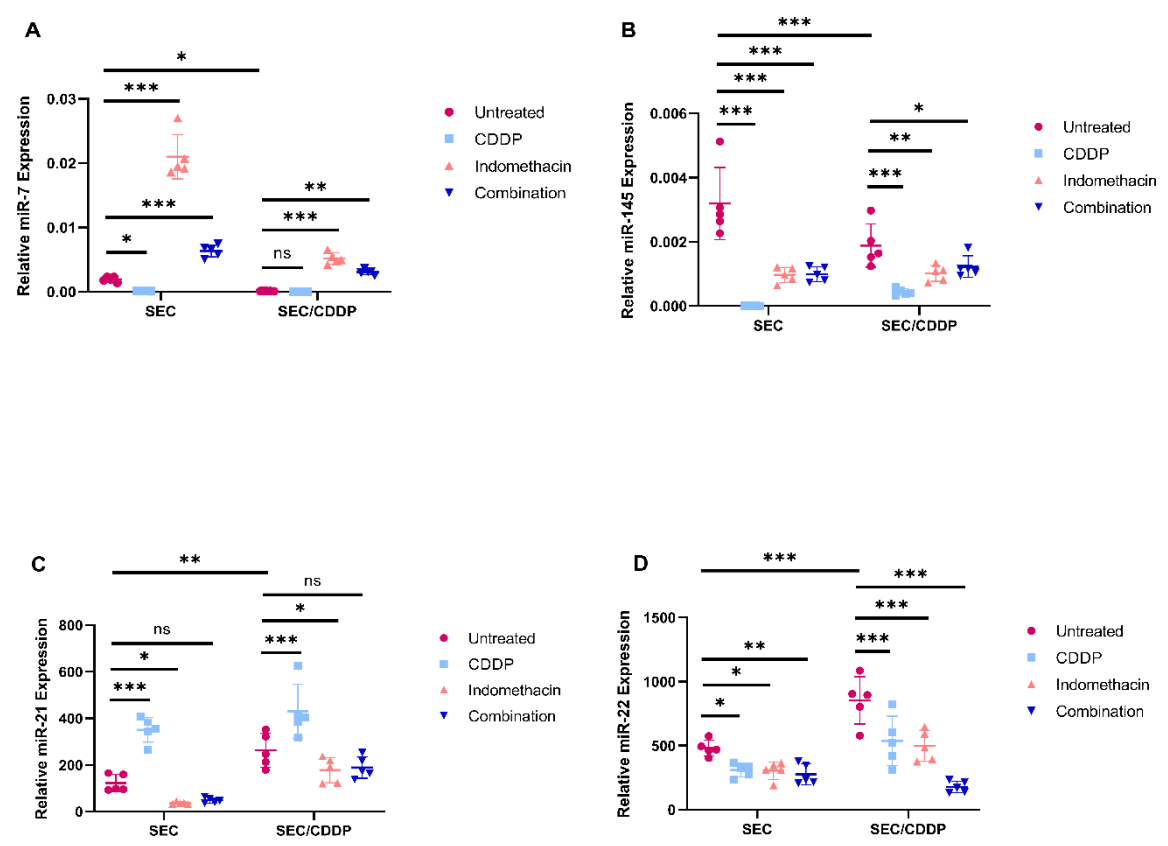

Fig. 4. Scatter plot of the expression patterns of microRNAs genes analyzed by qRT-PCR. Two-way ANOVA analysis was done $(\mathrm{n}=5)$ A. Relative miR-7 expression. B. Relative miR-145 expression. C. Relative miR-21 expression. D. Relative miR-22 expression. Data are expressed as mean \pm SD. ${ }^{*} p$ $<0.05,{ }^{* *} p<0.01,{ }^{* *} p<0.001, \mathrm{~ns}=$ nonsignificant. miR: microRNA, qRT-PCR: quantitative reverse transcription PCR, SEC: solid Ehrlich carcinoma, CDDP: cisplatin, SEC/CDDP: CDDP-resistant SEC. 


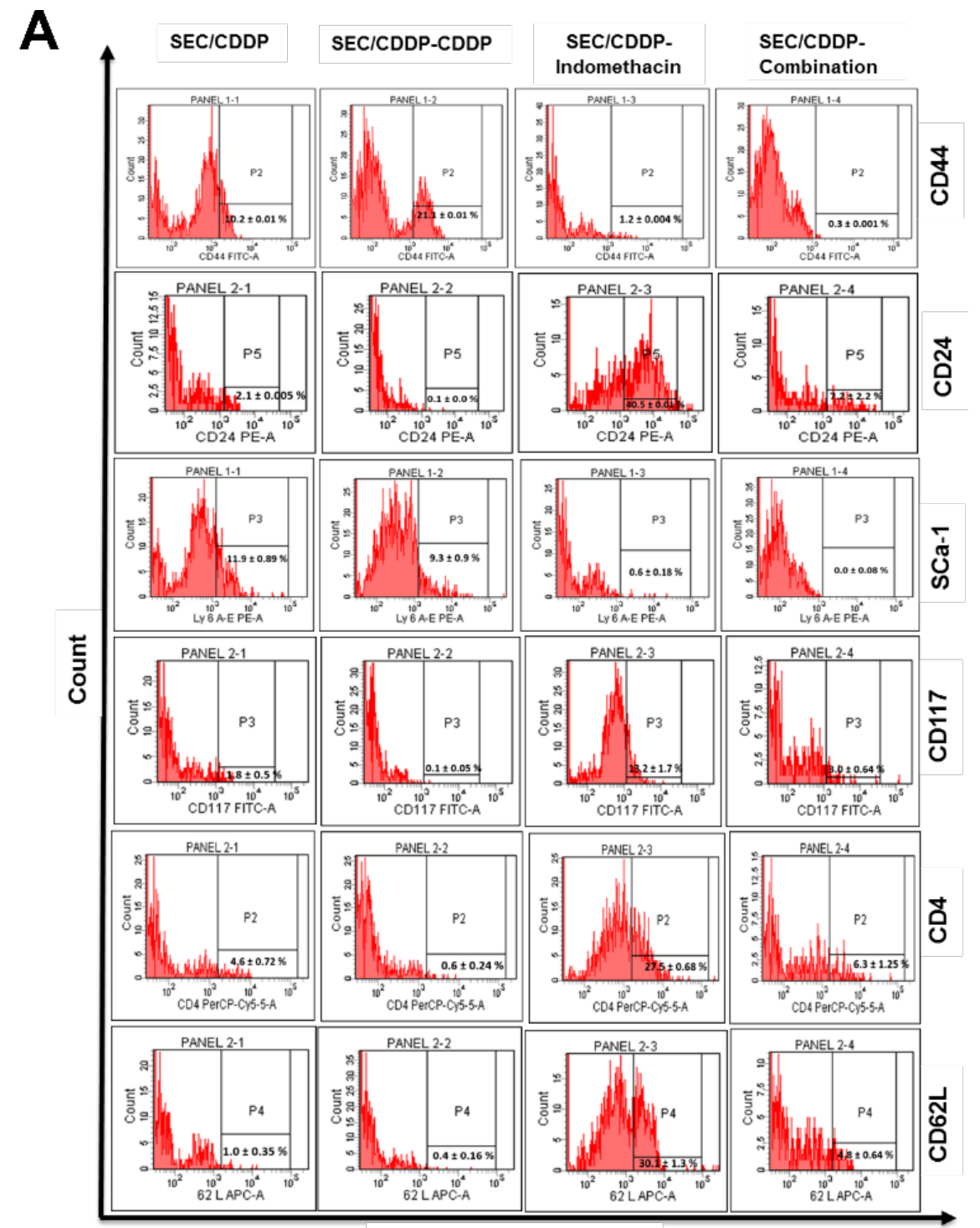

Fluorescence Intensity

B

Fig. 5. Flow cytometry of the BCSCs and immunological markers . A. Histograms of flow cytometric analysis of CSCs markers; CD44, CD24, and SCa-1 as well as immune cells markers; CD117, $\mathrm{CD} 62 \mathrm{~L}$, and CD4. Blood samples were collected from mice in the SEC/CDDP groups. B. One-way ANOVA was done $(\mathrm{n}=5)$. Data are expressed as mean $\pm \mathrm{SD} .{ }^{*} p<0.05,{ }^{* *} p<0.01,{ }^{* * *} p<0.001$, ns $=$ nonsignificant. BCSCs: breast cancer stem cells, CD: cluster of differentiation, SCa-1: stem cell antigen 1, SEC: solid Ehrlich carcinoma, SEC/CDDP: cisplatin-resistant SEC, SEC/CDDP-CDDP: cisplatin-resistant SEC treated with cisplatin, SEC/CDDP-indomethacin: cisplatin-resistant SEC treated with indomethacin, SEC/CDDP-combination: cisplatin-resistant SEC treated with CDDP and indomethacin.
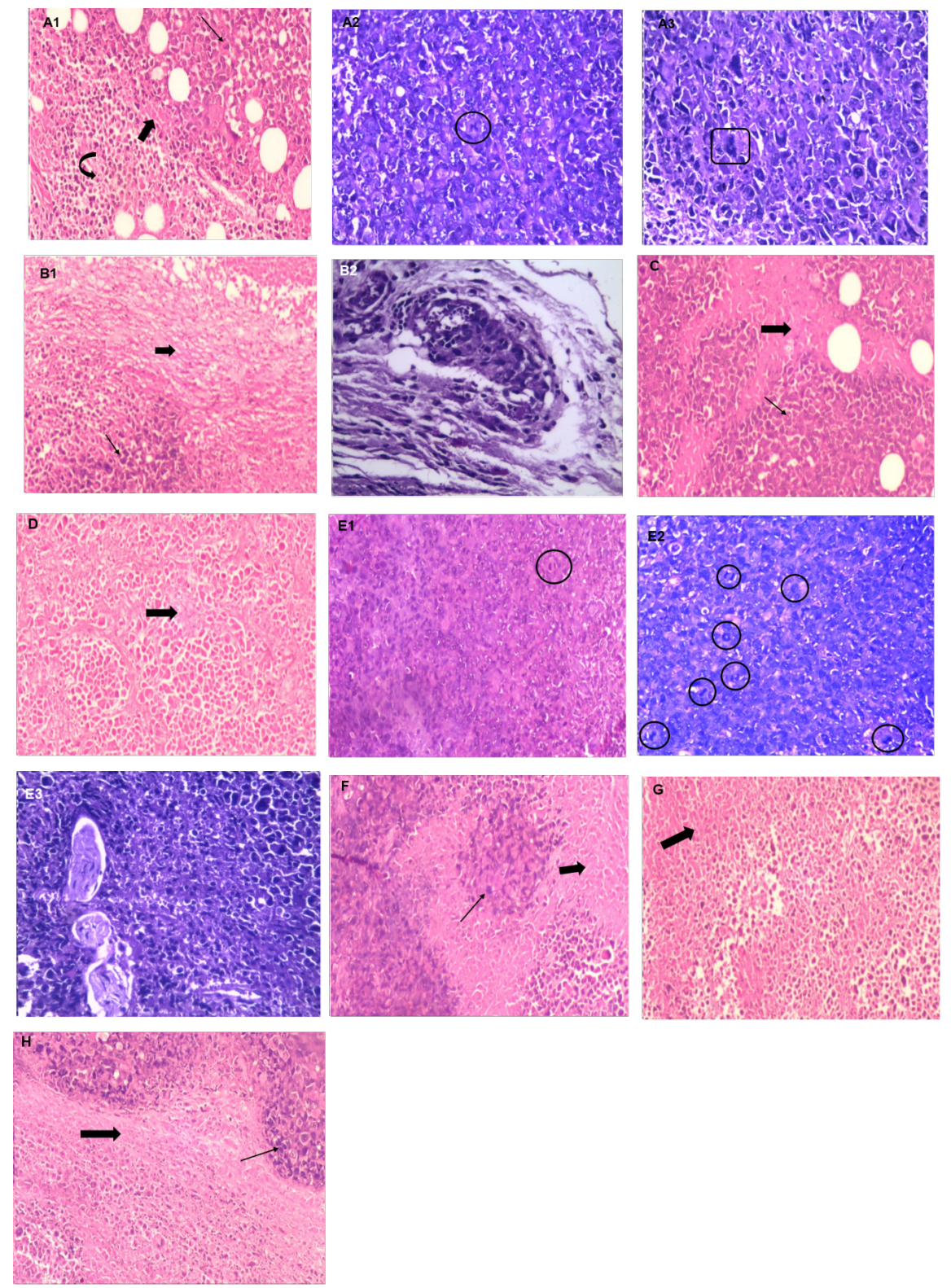

Fig. 6. $\mathbf{H} \& \mathrm{E}$ staining of tumor sections under $\times \mathbf{2 0 0}$ microscope. The interpretation was made by two pathologists which accounts for the color difference due to different microscopes used.

A1: SEC tumors showing cells with pleomorphism, hyperchromatism and high N/C ratio admixed with few 
areas of necrosis and apoptotic bodies.

A2: SEC tumors showing mitosis. A3: SEC tumors showing bizarre giant tumor cells.

B1: SEC-CDDP showing extensive areas of necrosis, however viable tumor cells could be detected.

B2: SEC-CDDP forming a vascular embolus.

C: SEC-indomethacin showing little effect of indomethacin with still viable tumor cells with few areas of necrosis.

D: SEC-combination showing extensive areas of necrosis [ghost cells] with no viable tumor cells

E1: SEC/CDDP showed very aggressive tumor cells with extensive mitosis, no necrosis or apoptotic bodies could be detected.

E2: A wide field of mitosis with pleomorphism in SEC/CDDP tumor sections.

E3: Perineural invasion in SEC/CDDP tumor tissues.

F: SEC/CDDP-indomethacin showing viable tumor cells with few areas of necrosis and apoptotic bodies

G: SEC/CDDP-combination showing extensive areas of necrosis [ghost cells] with very few viable tumor cells.

H: SEC/CDDP-CDDP showing extensive areas of necrosis, however, viable aggressive tumor cells could be detected. 